\title{
Tecnología de sensores inalámbricos para el manejo del ferti- riego en el viñedo en Galicia
}

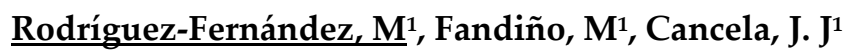

$1 \quad{ }^{1}$ GI-1716. Proepla. Depart. Ingeniería Agroforestal. Escuela Politécnica Superior de Ingeniería. Universidad de Santiago de Compostela: marta.rodriguez.fernandez0@rai.usc.es; maria.fandino@usc.es; javierjose.cancela@usc.es

Resumen: Actualmente, la gestión eficiente del uso del agua en el viñedo, es una tendencia ya implantada en el mundo vitivinícola, que cada día avanza ligada a las nuevas tecnologías, de cara a mejorar la eficiencia del riego de la vid.

En los últimos años, la tecnología inalámbrica ha revolucionado la forma de ver y comprender el manejo del viñedo, eliminando restricciones y dando más flexibilidad a la hora de implantar tecnologías sobre la viña. Gracias a esta evolución tecnológica, se han desarrollado sensores inalámbricos (WSN), que posibilitan la obtención de datos en cualquier localización a tiempo real.

En el presente estudio, se analiza la gestión del riego de un viñedo situado en Vedra (A Coruña), en las campañas 2019 y 2020. En la parcela a estudio, se encuentran instalados sensores Watermark ${ }^{\mathrm{TM}}$, que miden el potencial de agua en el suelo cada hora, a 30 y $60 \mathrm{~cm}$ de profundidad. Con los datos disponibles de lluvia y evapotranspiración del viñedo, se ha realizado un análisis de la evolución del potencial foliar a lo largo de las campañas en estudio. El potencial hídrico foliar de tallo a mediodía solar, se utilizó como estimador del estado hídrico del viñedo, buscando correlaciones entre ambos potenciales: suelo y hoja.

En las condiciones del ensayo, las medidas tensiométricas a $30 \mathrm{~cm}$, pueden ser una herramienta útil para la monitorización del riego. Si bien, se plantean distintas alternativas y mejoras del sistema que permiten el manejo del riego de la vid dentro de la eficiencia en el uso del agua, que engloba la aplicación de una viticultura de precisión.

Palabras clave: tecnología inalámbrica; sensores Watermark ${ }^{\mathrm{TM}}$; gestión del agua; riego viñedo 


\title{
XXXVIII Congreso Nacional de Riegos CARTAGENA 2021
}

\section{Wireless sensor technology for fertigation management in the vineyard in Galicia}

\author{
Rodríguez-Fernández, $\mathbf{M}^{1}$, Fandiño, $\mathbf{M}^{1}$, Cancela, J. J ${ }^{1}$
}

1 GI-1716. Proepla. Ingeniería Agroforestal. Depart. Escuela Politécnica Superior de Ingeniería. University of Santiago de Compostela: marta.rodriguez.fernandez0@rai.usc.es; maria.fandino@usc.es; javierjose.cancela@usc.es

\begin{abstract}
Currently, the efficient management of water use in the vineyard, is a trend already established in the wine world, which progresses every day linked to new technologies, order to improve the efficiency of vine irrigation. Actually, wireless technology has revolutionized the point of view of vineyard management, removing restriction and giving more flexibility when implementing technologies in the vine. This technological evolution, wireless sensors (WSN) have been developed, which make it possible to obtain data in any location in real time.

In this study, the irrigation management of a vineyard located in Vedra (A Coruña) is analysed in 2019 and 2020 seasons. In the plot under study, Watermark ${ }^{\mathrm{TM}}$ sensors are installed for measure the water potential in the soil every hour, 30 and $60 \mathrm{~cm}$ deep. With the available data in rainfall and evapotranspiration of the vineyard, an analysis of the evolution of the foliar potential throughout the seasons has been carried out. The foliar hydric potential of the stem at solar noon was used as an estimator of the hydric state of the vineyard, looking for correlations between both potentials: soil and leaf.

Under study conditions, tensiometric measurements at $30 \mathrm{~cm}$, can be a useful tool for monitoring irrigation. However, different alternatives and improvements of the system allow the management of vine irrigation within the efficiency in the use of water, which includes the application of precision viticulture.
\end{abstract}

Keywords: wireless technology; watermark ${ }^{\mathrm{TM}}$; water management; vineyard irrigation 


\section{Congreso Nacional de Riegos CARTAGENA 2021}

\section{Introducción}

La tecnología de sensores inalámbricos, se ha instalado en el campo de la viticultura como una nueva tecnología que aporta información sobre el estado del viñedo a tiempo real, que permite realizar un manejo del cultivo de forma eficiente [1].

El manejo del agua en el cultivo de la vid, se considera una herramienta importante para mejorar el desarrollo de la planta y la calidad de la uva, así como para conseguir la estabilidad en la producción a final de campaña [2].

Una de las mayores demandas de los viticultores para manejar el riego, es conocer el estado hídrico del viñedo de modo rápido, que no implique excesivo consumo de tiempo y mano de obra, tal como requiere el potencial hídrico foliar, además de ser una medida destructiva [3]. El estado hídrico de la planta depende de su desarrollo foliar, de la demanda atmosférica y del contenido de agua en el suelo [4]. Por ello, en este trabajo se plantea como objetivo el elaborar y validar los sensores inalámbricos instalados en el viñedo como una nueva tecnología que aporte información sobre el estado hídrico de la planta a partir de la disponibilidad hídrica del suelo, contrastado con la demanda ambiental y el desarrollo foliar, disponible automáticamente y con posibilidad de tener la información actualizada en tiempo real.

\section{Materiales y métodos}

\subsection{Parcela a estudio}

El viñedo a estudio se ecuentra enmarcado en la Denominación de Origen Rías Baixas (ACoruña), concretamente perteneciente a la subzona vitivinícola de Ribeira de Ulla. La parcela tiene una extensión de 12,1 ha, situada a $42^{\circ} 45^{\prime} 6^{\prime \prime}$ longitud Norte, $8^{0} 28^{\prime} 10^{\prime \prime}$ longitud Oeste y a $131 \mathrm{~m}$ sobre el nivel del mar.

El viñedo ha sido plantado en el año 2004, en toda su extensión cultivado con la variedad 'Albariño', variedad preferente en las Rías Baixas. Las vides se encuentran empalizadas en espaldera y con un sistema de poda de pulgar-vara. La distancia entre plantas es de 2,5 m entre plantas y $3 \mathrm{~m}$ entre filas. El portainjerto empleado es el 110 Ritcher y dispone de riego por goteo en toda la superficie cultivada, con un riego de campaña gestionado a criterio del viticultor.

\subsection{Dispositivo experimental}

Los puntos de muestreo de la parcela a estudio, se seleccionaron siguiendo una malla de $30 \times 30 \mathrm{~m}$, que dio a lugar a 32 puntos de muestreo que recogen el área total del viñedo, permitiendo analizar la extensión total de la parcela, de manera homogénea en cada uno de los cinco sectores de riego que presenta el viñedo.

\subsection{Mediciones}

El contenido volumétrico del agua en el suelo del viñedo, se determinó mediante sondas inalámbricas Watermark ${ }^{\mathrm{TM}}$, los sensores instalados dentro de un tubo de acceso se encuentran a 30 y 60 $\mathrm{cm}$, conectados a un nodo central que registra datos automáticamente a cada hora y diariamente durante el período a estudio (campañas 2019 y 2020).

El potencial hídrico de tallo, se medió tres veces por campaña en los meses de junio, julio y agosto, a mediodía solar, utilizando la técnica de la cámara de presión, envolviendo las hojas en bolsas opacas una hora antes de la lectura del potencial, estas medidas se llevaron a cabo en hojas sanas y adultas, 


\section{Congreso Nacional de Riegos CARTAGENA 2021}

que se encontrasen expuestas a la radiación solar directa [5,6]. Se realizaron medidas en los 32 puntos de muestreo de la parcela.

También se han recogido datos meteorológicos en las dos campañas a estudio de la estación agroclimática Pazo Galegos, gestionada meteogalicia (https://www.meteogalicia.gal/web/inicio.action). La zona vitivinícola de las Rías Baixas, se encuentra integrada en la región Atlántica, donde la climatología de la zona, se define con inviernos protagonizados por borrascas del Oeste y del Sudoeste, con frentes cálidos y aire tropical, que recaen en unas precipitaciones abundantes y temperaturas suaves.

Para evaluar la relación entre el potencial hídrico de la planta y el contenido en agua del suelo, se promediaron los datos recogidos por las sondas de 30 y $60 \mathrm{~cm}$ en los días en los que se llevaron a cabo las medidas de potencial hídrico de tallo y se analizaron los datos utilizando métodos de correlación lineal.

\section{Resultados y discusión}

Con los resultados obtenidos del seguimiento de datos de los sensores en ambas campañas, ha podido realizarse un control en los meses de mayo a septiembre, que analizado junto con las condiciones meteorológicas que se han dado en el período a estudio, ha podido observarse cómo en ambas campañas el requerimiento de agua en el suelo es mayor en los meses de julio-agosto (2019) y abriljulio (2020), coincidente con un período en los que las precipitaciones son escasas y focalizadas en días en los que se atenúan los picos medidos del potencial hídrico en el suelo.

Si bien, también puede observarse como la diferencia de los datos generados con los sensores instalados a 30 y $60 \mathrm{~cm}$ del suelo, a menor profundidad $(30 \mathrm{~cm})$ se observa una tendencia hídrica del suelo más estable, ascendente desde el mes de junio (2019) y abril (2020) hasta el mes de septiembre, por otro lado, los datos recogidos a $60 \mathrm{~cm}$ de profundidad, en la campaña 2019 registra valores ligeramente inferiores a $60 \mathrm{kPa}$, con picos focalizados en los meses de junio y septiembre, mientras que en la campaña 2020 estos sensores han presentado un brusco ascenso en el mes de abril y un fallo en la conexión de la generación de los datos del mismo en los meses de junio y agosto (Figuras 1 y 2 ).

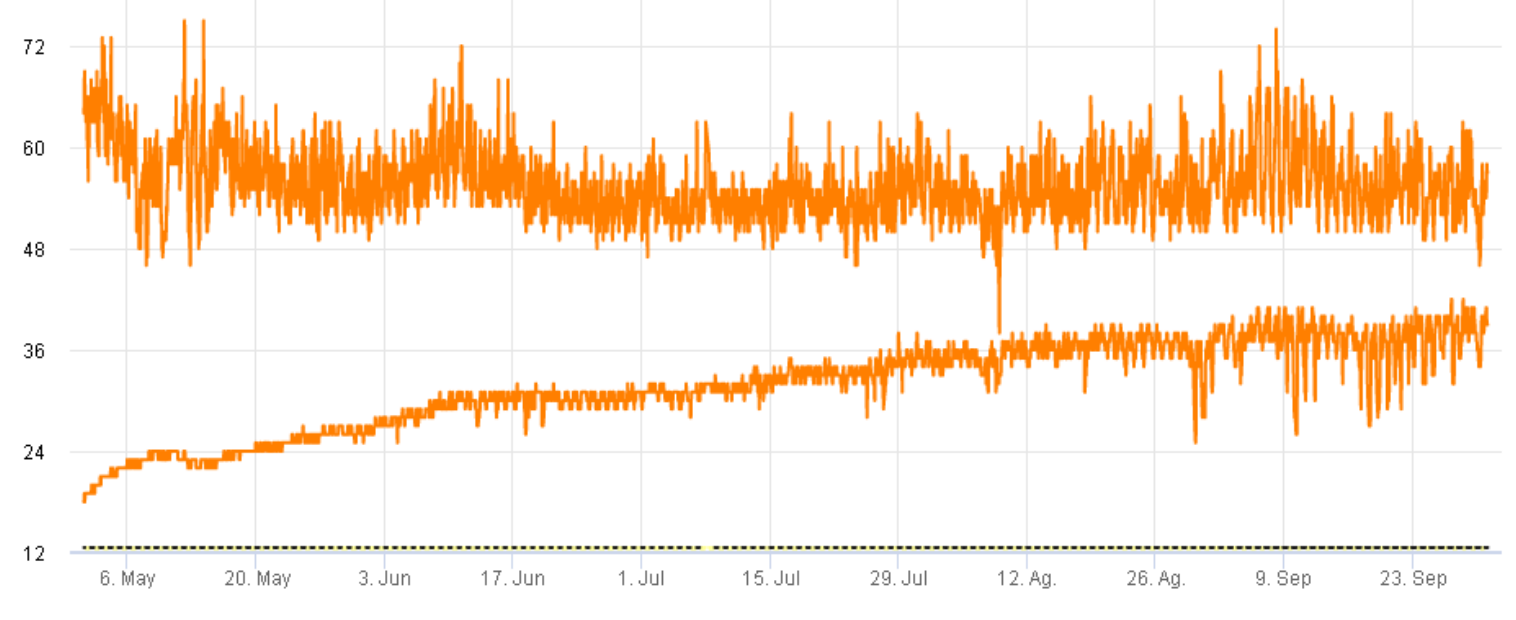




\section{Congreso Nacional de Riegos CARTAGENA 2021}

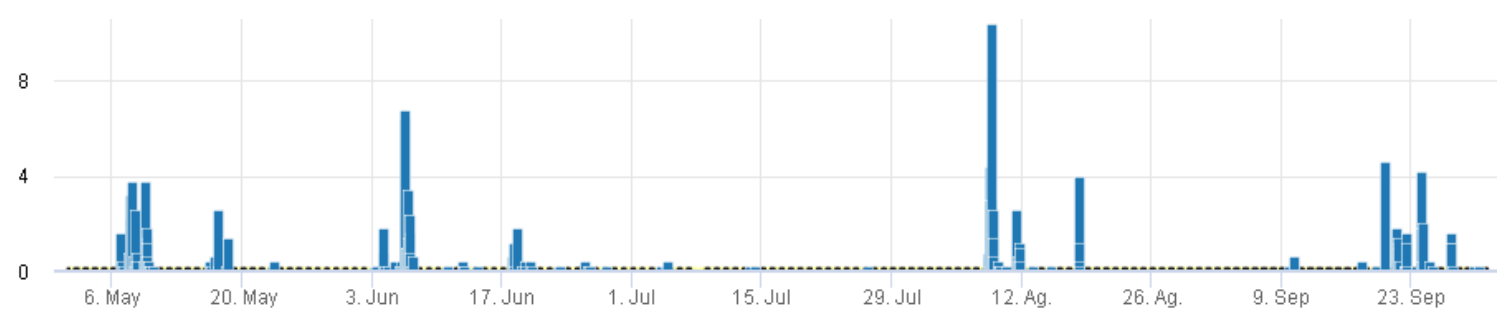

Figura 1. Registro de datos de las sondas de sensores de potencial hídrico de suelo $(\mathrm{kPa})$ a 30 y $60 \mathrm{~cm}$ de profundidad y precipitación diaria (mm)(mayo-septiembre 2019)

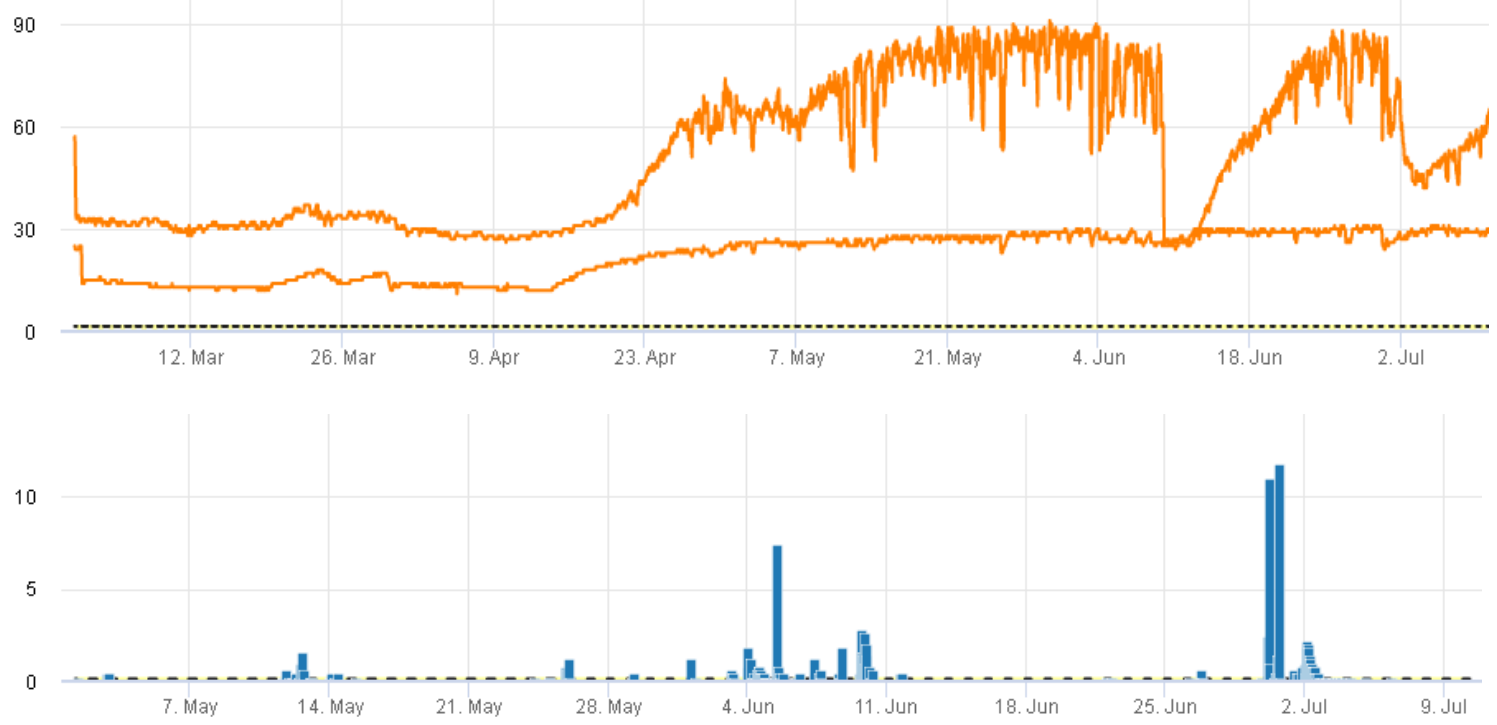

Figura 2. Registro de datos de las sondas de sensores de potencial hídrico de suelo $(\mathrm{kPa})$ a 30 y $60 \mathrm{~cm}$ de profundidad y precipitación diaria (mm) (mayo-julio 2020)

Con las relaciones lineales realizadas entre el potencial de suelo y las mediciones de potencial hídrico de tallo, se ha establecido que los sensores instalados a $30 \mathrm{~cm}$ de profundidad, son los que mejor se ajustan a las medidas realizadas en campo, con valores de $r^{2}: 0,9$ en la campaña 2019 y r²: 0,8 en 2020. Los sensores instalados a $60 \mathrm{~cm}$, también han presentado buenas relaciones, no obstante, en este caso en la campaña 2019 se ha obtenido un valor de correlación de 0,9, mientras que en 2020 este valor descendió a 0,4 influenciado por los problemas de conexión que se han dado a lo largo de la campaña a estudio (Figura 3). 

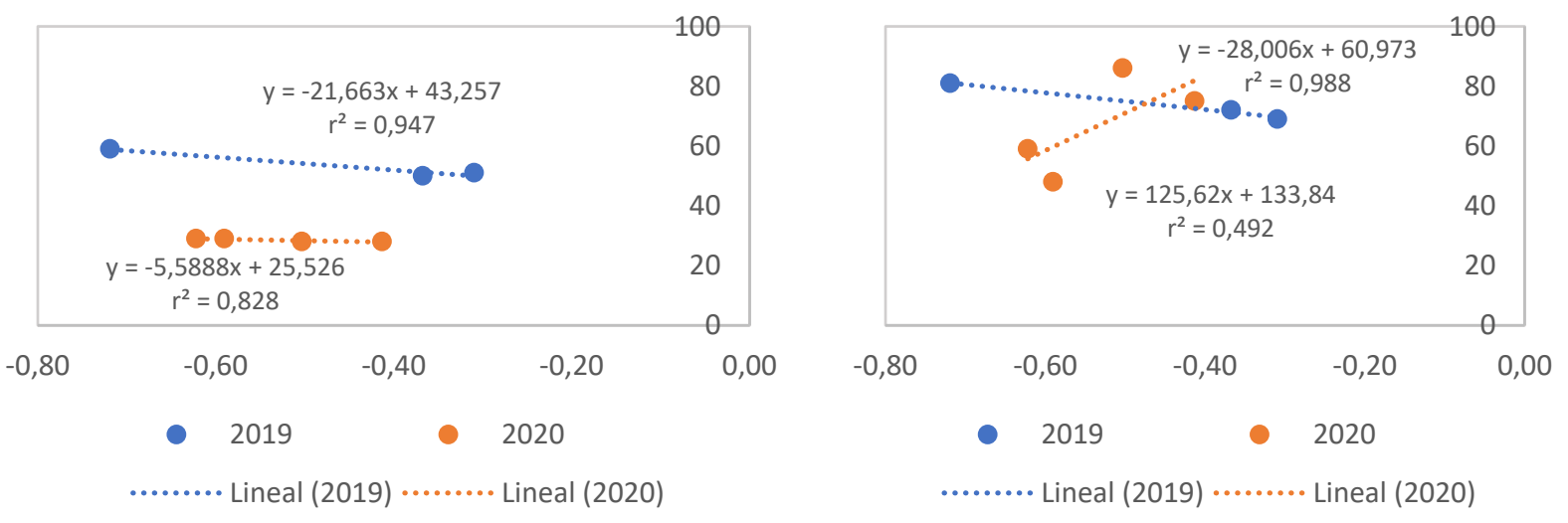

Figura 3. Relaciones lineales entre potencial hídrico de suelo $(\mathrm{kPa})$ y potencial hídrico foliar $(\mathrm{MPa})$, sensores a $30 \mathrm{~cm}$ de profundidad representados a la izquierda y sensores a $60 \mathrm{~cm}$ de profundidad representados a la derecha.

Estas relaciones determinadas entre el potencial hídrico de suelo y el potencial hídrico de tallo, también han sido estudiadas para la misma variedad 'Albariño' por otros autores [7], obteniendo también buenas relaciones entre ambas medidas.

El estudio de los sensores a distintas profundidades, también han sido analizados por distintos autores, que obtienen buenos resultados con sensores instalados en los 30 primeros $\mathrm{cm}$ del suelo [8,9]. Por otro lado, hay estudios que apuntan a que las medidas de contenido de humedad del suelo en sistemas de riego localizado, no son representativas en los primeros $\mathrm{cm}$ de suelo, debido a que el consumo de agua es más rápido que en las capas más profundas del mismo, por lo que, en este caso se considera más fiable tomar estos datos de potencial hídrico de suelo, en un rango de $60 \mathrm{~cm}$ de profundidad [10].

\section{Conclusiones}

Las conclusiones obtenidas en el presente estudio, muestran muy buenas relaciones entre los valores de potencial hídrico foliar y el potencial de agua en el suelo, evaluados mediante sensores inalámbricos instalados en el viñedo a 30 y $60 \mathrm{~cm}$ de profundidad. Estos valores obtenidos, muestran como los sensores instalados Watermark ${ }^{\mathrm{TM}}$, son una tecnología muy útil para determinar el potencial de agua en el viñedo a lo largo de la campaña, que permite la aplicación y control del riego de una forma más eficiente.

Sin embargo, las medidas analizadas en este trabajo, deben de replicarse en cuanto a campañas y profundidades de los sensores, que permitan determinar un rango mucho más estrecho en cuanto a la colocación óptima de los sensores en el viñedo. Siendo necesario ampliar la variabilidad del rango de mediciones del potencial de suelo, limitado en el estudio a valores entre 20 y $80 \mathrm{kPa}$.

\section{Referencias}

1. Blackmore, S. Precision farming: and introduction, Outlook Agr., 1994; pp. 275-280.

2. Williams, L.E., Matthews, M.A. Grapevine. In: Stewart BA, Nielsen DR (eds) Irrigation of agricultural crops agronomy monograph no. 30. ASA-CSSA-SSSA, Madison. 1990. pp 1019-1055.

3. Baena, M., Rodríguez, L., Tejero, M., Baeza, P. Estimation of the hydric state of the vineyard from the water content of the soil, environmental conditions and foliar development. In II Conference on viticulture: technical communications: Madrid, November 2016. Pp. 236-242. Polytechnic University of Madrid. 
4. Williams, L.E., Baeza, P. Relationships among ambient temperature and vapour pressure deficit and leaf and stem water potentials of fully irrigated, field-grown grapevines. American Journal of Enology and Viticulture. 2007. 58 (2) pp. $173-181$.

5. Scholander, P.H., Hammel, H.J., Bradstreet, A., Hemmingsen, E.A. Sap pressure in vascular plants. Science. 1965 pp. $339-$ 346.

6. Choné, X., Van Leeuwen, C., Dubordieu, D., Gaudillère, J.P. Stem water potential is a sensitive indicator of grapevine water status. Annals of Botany, 2001 pp. 447-483.

7. Martínez, E.M., Fandiño, M., Cancela, J.J., Rey, B.J. Evaluation of the stress of the cv. Albariño in the DO Rías Baixas. Digital magazine Interempresas Wine Industry. 2013.

8. Williams, L.E., Araujo, F.J. Correlations among predawn leaf, midday leaf, and midday stem water potential and their correlations with other measures of soil and plant water status in Vitis vinifera J. Amer. Soc. Hrtic.Sci. 2002 pp.448-454.

9. Asenjo, J.L., Yuste, J. Estimation of the hydric state of the soil bytensiomety and volumetry and its relationship with the hydric state of the vineyard. Studies of the unsaturated zone of the soil. 2003.

10. Bravdo, B., Proebsting, E.I. Use of drip irrigation in orchards. HorTechnology. 1993 pp. 44-49. 